\title{
Does PFNA II Avoid Lateral Cortex Impingement for Unstable Peritrochanteric Fractures?
}

\author{
George A. Macheras MD, PhD, Stefanos D. Koutsostathis MD, PhD, \\ Spyridon Galanakos MD, Konstantinos Kateros MD, PhD, \\ Stamatios A. Papadakis MD, PhD
}

Published online: 4 July 2012

(C) The Association of Bone and Joint Surgeons (B) 2012

\begin{abstract}
Background Proximal femoral nail antirotation devices (PFNAs) are considered biomechanically superior to dynamic hip screws for treating unstable peritrochanteric fractures and reportedly have a lower complication rate. The PFNA II was introduced to eliminate lateral cortex impingement encountered with the PFNA. However, it is unclear whether the new design in fact avoids lateral cortex impingement without compromising stability of fixation and fracture healing.

Questions/Purposes We therefore asked whether the PFNA II: (1) eliminates the lateral cortex impingement and fracture displacement experienced with PFNA; and (2) provides stable fracture fixation with a low major complication rate for unstable fractures in European patients.
\end{abstract}

Each author certifies that he or she, or a member of their immediate family, has no commercial associations (eg, consultancies, stock ownership, equity interest, patent/licensing arrangements, etc) that might pose a conflict of interest in connection with the submitted article

Clinical Orthopaedics and Related Research neither advocates nor endorses the use of any treatment, drug, or device. Readers are encouraged to always seek additional information, including FDA-approval status, of any drug or device prior to clinical use. All ICMJE Conflict of Interest Forms for authors and Clinical Orthopaedics and Related Research editors and board members are on file with the publication and can be viewed on request.

Each author certifies that his or her institution approved the human protocol for this investigation, that all investigations were conducted in conformity with ethical principles of research, and that informed consent for participation in the study was obtained.

G. A. Macheras $(\square)$, S. D. Koutsostathis, S. Galanakos,

K. Kateros, S. A. Papadakis

4th Department of Orthopaedics and Traumatology, KAT

Hospital, 2 Nikis Street, 14561 Athens, Greece

e-mail: gmacheras@gmail.com
Methods We retrospectively reviewed 108 patients with an unstable peritrochanteric fracture, 58 treated with PFNA and 50 with PFNA II. We compared nail positioning, major and minor complication rates, operative and fluoroscopy time, blood transfused, time to mobilization, hospital stay, fracture union, and Harris hip score. The minimum followup was 12 months (mean, 13 months; range, 1218 months).

Results In the PFNA II group we encountered no impingement on the lateral cortex and no patients with lateral fragment or loss of reduction at insertion, whereas with the PFNA group, we had 10 and five cases, respectively. Fracture union occurred in all patients treated with PFNA II without mechanical failures. PFNA II cases were associated with a slightly shorter surgical time than PFNA cases (23 minutes versus 27 minutes, respectively).

Conclusion PFNA II avoided lateral cortex impingement experienced with PFNA, providing fast and stable fixation of the unstable peritrochanteric fractures.

Level of Evidence Level III, retrospective comparative study. See Guidelines for Authors for a complete description of levels of evidence.

\section{Introduction}

Increased life expectancy has led to a considerably increased incidence of proximal femoral fractures [17]. The standard of peritrochanteric fracture treatment is stable fixation, which allows early full weightbearing mobilization of the patient $[1,5,10,24,27]$. The choice of surgical treatment is determined in part by whether the fracture is judged stable or unstable. Unstable intertrochanteric fractures are those with major disruption of the posteromedial cortex because of comminution or are fractures with 
reverse oblique or subtrochanteric fracture lines. Fractures without posteromedial cortex disruption or subtrochanteric extension are considered stable $[25,26]$.

For stable fracture types (eg, A1 AO/OTA classification [25]), sliding or dynamic hip screw systems provide stable fixation with a low major complication rate [9]. In patients with unstable fractures (eg, A2, A3 AO/OTA classification), dynamic hip screws (DHSs) have been associated with increased complication rates such as cutout of the head screw [31]. In these cases with cortical comminution, progressive displacement is more likely to occur using a DHS compared with an intramedullary nail $[34,35]$. The most common failure mechanism is migration of the femoral head into varus and retroversion and subsequent extrusion, or so-called cutout, of the lag screw through the femoral head [43]. Migration can also occur by concomitant femoral head rotation around the implant shaft [35]. These phenomena are most likely attributable to an insufficient purchase of the implant in the femoral neck, especially in the presence of predisposing factors such as osteoporosis [20] or poor reduction [6]. For these patients, several intramedullary nails have been developed $[12,28$, 29] that are reportedly superior biomechanically [8, 32]. Positioning of the intramedullary device close to the weightbearing axis of the femur reduces forces on the implant. The primary innovation of the proximal femoral nail antirotation (PFNA, AO/ASIF) design is the helical neck blade that reduces the risk of bone loss and offers improved purchase in the femoral head as a result of compaction of cancellous bone around the blade during insertion [35, 36, 41]. Rotation of the head-blade combination as a whole is prevented by an intrinsic locking mechanism [3, 38]. Furthermore, given controlled impaction of the metaphyseal fracture zone, immediate full weightbearing is allowed. Despite the fact that PFNA provides high union rates with low major complication rates [13, 14, 42], it has been associated with lateral cortex impingement [40] that causes lateral cortex fracture and fracture displacement during insertion. The round profile of the nail creates pressure to the lateral wall and the headneck fragment (Fig. 1), thus damaging the lateral wall and causing loss of reduction and varus of the head-neck fragment, a complication that decreases stability and increases the risk to cutout.

The PFNA II was designed to overcome those concerns. Its mediolateral angle is reduced to $5^{\circ}$, allowing a slightly more lateral entry point through the tip of the greater trochanter. Furthermore, it has a more flattened lateral surface (Fig. 2) that theoretically decreases the length of the region of impingement on the lateral cortex (Fig. 3) thus reducing the risk of fracture during insertion. Lv et al. [23] recently reported that proximal femoral nail antirotation for unstable trochanteric fractures in Asian patients provided an

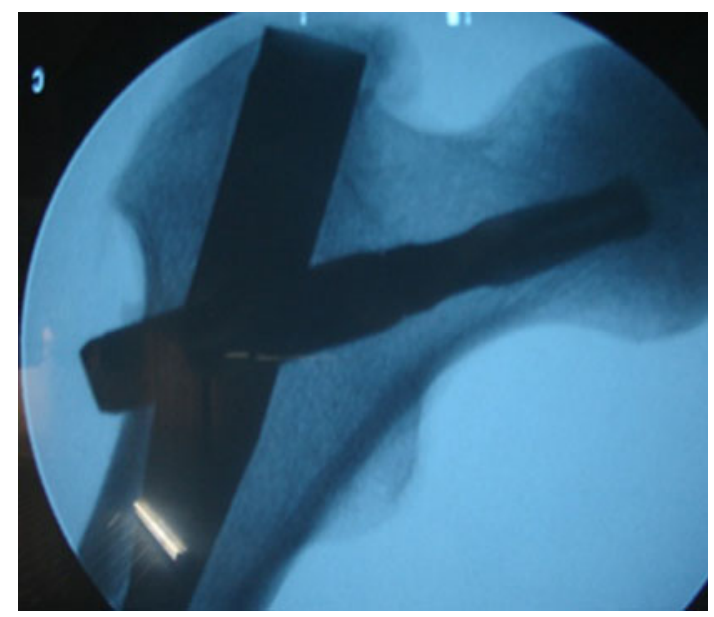

Fig. 1 This figure illustrates an intertrochanteric fracture treated with the use of PFNA. Note the impingement of the nail to the lateral cortex of the femur pointed with a white arrow.
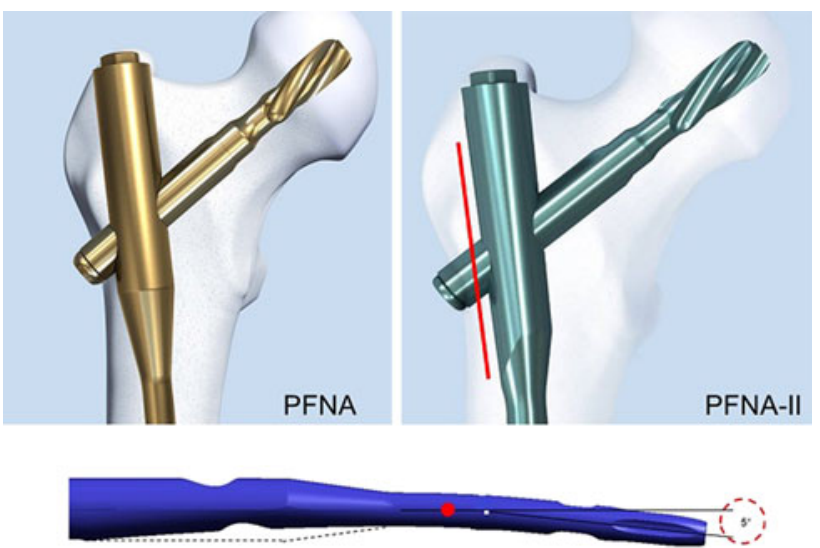

PFNA-II

Fig. 2 PFNA is proximally rounded in contrary to PFNA II that has a more flattened lateral surface. The mediolateral angle of PFNA II is decreased to $5^{\circ}$.

anatomic fit in the proximal femur with $95 \%$ ideal position of the nail and eliminated complications related to fixation. Tyagi et al. [39] analyzed the geometric discrepancies between the proximal femur and two types of PFNA (PFNA and PFNA II) using CT-based analysis in Asian patients. They concluded the morphological incompatibility between the proximal femur and PFNA originated from the difference in the bending angle on the coronal section and the angle of inclination of the lateral cortex and found the flat lateral shape of PFNA II lessened impingement between the lateral side of the proximal femoral nail and the lateral cortex of the proximal femur.

To confirm these findings we asked whether the PFNA II: (1) eliminated the lateral cortex impingement and fracture displacement experienced with PFNA; and (2) provided stable fracture fixation with a low major complication rate for unstable fractures in European patients. 


\section{Patients and Methods}

Between January 2009 and September 2010, we treated 130 patients with unstable fractures of the proximal part of the femur (31A2, 31A3). The first 72 patients were treated with PFNA; this nail was replaced by PFNA II in our practice for the next 58 cases between February 2010 and September 2010 because we were asked by Synthes (Solothurn, Switzerland) to test the new nail design (PFNA II) regarding the occurrence of lateral cortex impingement, major complications, and fracture healing in unstable fracture types. We excluded 13 patients because of the presence of one or more of the following exclusion criteria: age younger than 60 years, multiple trauma, open injuries, pathological fracture, or a severe medical condition. Another nine patients died or were lost to followup. Of the remaining 108 patients, 58 were treated with PFNA and 50 with PFNA II. Sixty-one were females and 47 were males.

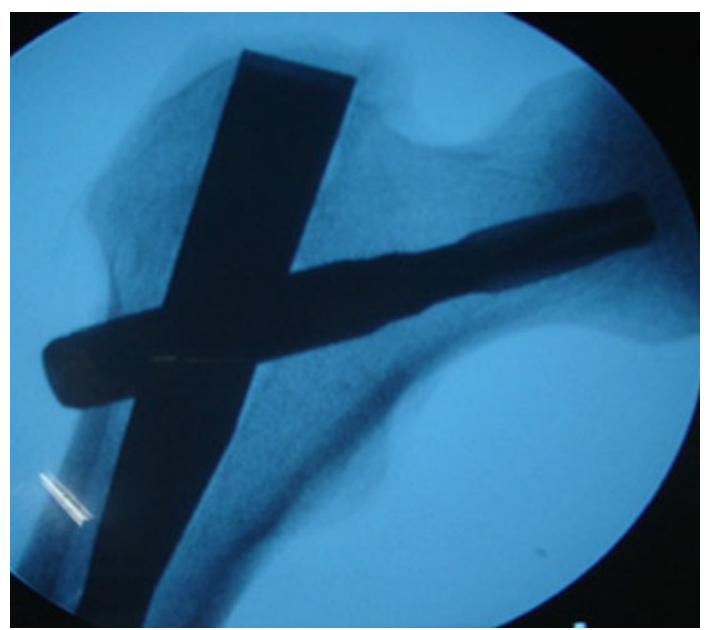

Fig. 3 This figure illustrates an intertrochanteric fracture treated with the use of PFNA II. The arrow points to the lack of impingement between the flattened lateral surface of the nail and the lateral femoral cortex.
The mean age was 78.2 years (range, $60-93$ years). The right hip was involved in 59 of the 108 patients. Each fracture was classified using the AO/ASIF classification [25]. The minimum followup was 12 months (mean, 13 months; range, 12-18 months). No patients were recalled specifically for this study; all data were obtained from medical records and radiographs.

Details of all patients were retrieved from their files, operative reports, and regular followup visits. We recorded demographic data and comorbid medical conditions (Table 1) and type of injury (Table 2). The two groups had similar demographics and comorbidities (Table 1).

Operations were performed by one of three experienced orthopaedic trauma surgeons (GAM, SDK, SAP) familiar with the technique of extra- and intramedullary nailing of the proximal femur. All patients had spinal anesthesia. All operations were performed on a radiolucent traction table where closed reduction of the fracture was carried out under image intensification. When closed reduction failed, a limited open reduction was performed and a retractor was used for the elevation of the distal fragment with particular emphasis on the posteromedial wall reconstruction. In 13 cases, open reduction was necessary, eight in the PFNA group and five in the PFNA II group. A standard operative

Table 1. Demographics and comorbidities

\begin{tabular}{llll}
\hline $\begin{array}{l}\text { Demographic } \\
\text { or comorbidity }\end{array}$ & PFNA & PFNA II & p value \\
\hline Age & $78.09 \pm 7.76$ & $78.30 \pm 8.13$ & 0.889 \\
Sex (male/female) & $25(43) / 33(57)$ & $22(44) / 28(57)$ & 1.000 \\
Hypertension (yes) & $15(25.9)$ & $14(28.0)$ & 0.831 \\
Diabetes (yes) & $10(17.2)$ & $10(20.0)$ & 0.706 \\
Coronary disease (yes) & $8(13.8)$ & $8(16.0)$ & 0.791 \\
Parkinson's disease (yes) & $2(3.4)$ & $1(2.0)$ & 1.000 \\
\hline
\end{tabular}

All qualitative variables are presented as number $(\%)$; PFNA $=$ proximal femoral nail antirotation.

Table 2. Fracture and operation variables

\begin{tabular}{|c|c|c|c|}
\hline Variable & PFNA & PFNA II & $\mathrm{p}$ value \\
\hline Time to operation [median (minimum-maximum)] & $3.0(1-8)$ & $3.0(1-7)$ & 0.880 \\
\hline Mobility status (assisted/frame/unrestricted) & $1(2) / 5(9) / 52(90)$ & $2(4) / 4(8) / 44(88)$ & 0.771 \\
\hline Fixation (accepted/not accepted) & $53(91) / 5(9)$ & $45(90) / 5(10)$ & 1.000 \\
\hline Quality of reduction (excellent/good/accepted) & $31(53) / 17(30) / 10(17)$ & $30(60) / 12(24) / 8(16)$ & 0.775 \\
\hline Means of reduction (closed/open) & $51(88) / 7(12)$ & $44(88) / 6(12)$ & 1.000 \\
\hline Type of fracture A22-A23-A31-A32-A33 (AO classification) & $35-24-12-7-22$ & $40-24-12-6-18$ & 0.972 \\
\hline Nail impingement to the lateral cortex (yes) & $10(17.2)$ & $0(0)$ & 0.002 \\
\hline Lateral wall fragment (yes) & $5(8.6)$ & $0(0)$ & 0.076 \\
\hline Loss of reduction (yes) & $3(5.2)$ & $0(0)$ & 0.247 \\
\hline
\end{tabular}

All qualitative variables are presented as number (\%) except type of fracture, which is presented as percent; PFNA = proximal femoral nail antirotation. 
technique recommended by the manufacturer, as described in detail in instruction manuals, was used. The nail was inserted after a minimal approach proximally to the tip of the greater trochanter without canal reaming except from proximal 17-mm reaming. The blade was inserted without drilling. Distal interlocking was performed in a static manner in all cases. The nails used were $200 \mathrm{~mm}$ or $240 \mathrm{~mm}$ in length and $10 \mathrm{~mm}$ or $11 \mathrm{~mm}$ in diameter. Suction drains were not used in either of the PFNA groups. Bone grafting was not used in any of the 108 cases.

All patients received three doses of a prophylactic antibiotic regimen starting 2 hours before the operation. Suction drains were not used in any patients. The length of the operation from skin incision to skin closure and total radiation time were recorded. Criteria for transfusion were constant throughout the study period: hemoglobin value below $8 \mathrm{~g} / \mathrm{dL}$ or below $10 \mathrm{~g} / \mathrm{dL}$ in patients with coronary artery disease.

Low-molecular-weight heparin was routinely administered in all patients starting from admission and for a 6-week period. The rehabilitation protocol was uniform regardless of the fracture pattern and the type of fixation. Patients were encouraged to walk with a frame, allowing full weightbearing as tolerated, starting from the first postoperative day when capable. They were all encouraged to perform hip and knee exercises. We recorded time to mobilization, number of units of blood transfused, and length of hospitalization.

All patients were followed up at 1, 3, and 6 months and 1 year with physical examination of the affected limb. Plain AP and lateral radiographs were taken at each visit, incorporated into the patient's medical file, and reviewed for fracture union or implant failure. We classified walking ability into three categories: able to walk independently without aids or with a stick, walking independently with the help of aids (crutches or frame), and walking only when assisted by another person. Additionally, peri- and postoperative complications, need for transfusion, duration of surgery, fluoroscopy time, time to mobilization, time to discharge, and plain radiography data were all collected and recorded in detail. Potential complications were distinguished as (1) major: cutout, breakage, or migration of the implant, reoperation for any reason, pulmonary embolism, acute coronary infract, acute respiratory distress; and (2) minor, including wound healing disturbances, superficial wound infection, urinary tract infection, and superficial vein thrombosis [13]. Hip function was estimated with the Harris hip score [16]. There were no missing data through the followup period.

Plain AP and lateral radiographs were obtained on the first postoperative day and were retrospectively examined by one of us (GAM) specifically for this review for reduction of the fracture and position of the implant. One of us (GAM) evaluated all radiographs for quality of reduction and implant positioning, presence of impingement between the nail and lateral cortex, implant migration, cutout, and presence of callus on AP and lateral radiographs. The reduction was regarded as excellent if it was anatomical, good if it was up to $5^{\circ}$ of varus-valgus on the AP view or $5^{\circ}$ of ante- or retroversion on the lateral view and up to $5 \mathrm{~mm}$ of translation between the main fragments, acceptable if it was up to $10^{\circ}$ of varus-valgus on the AP view or $10^{\circ}$ of ante- or retroversion on the lateral view, and up to $10 \mathrm{~mm}$ of translation between the main fragments and unacceptable beyond those limits. Positioning of the nail into the femoral canal was evaluated, identifying cases in which impingement on the lateral cortex occurred on the AP view. We determined whether the blade was centrally located on both AP and lateral views. Fracture union was determined radiographically as the appearance of a bridging callus on three or four cortices on the AP and lateral views and clinically as a lack of pain sensation around the fracture site [15, 28].

The quantitative and qualitative variables are represented by the number of patients $(\mathrm{N})$, mean value (mean), $\mathrm{SD}$, median, interquartile range (IQR), and the frequencies (n) and percentages, respectively. The KolmogorovSmirnov test was used for normality analysis of the quantitative data. We determined differences in nail impingement to the lateral cortex, lateral wall fragment, loss of reduction, union, complications related to fixation, and systematic complication using the Fisher's exact test. The Mann-Whitney test was used to determine differences in the operative time between the two groups. We determined differences in mobility status, quality of reduction, and type using the chi square test. Fisher's exact test was also used to compare sex, concomitant diseases, and means of reduction. The differences in age, Harris hip score, and blood units transfused were determined using the independent samples t-test. The Mann-Whitney test was also used to compare time to operation, time to mobilization, and hospital stay. The Welch test was used in case of violation of equal variances (fluoroscopy time). All tests were two-tailed. All analyses were carried out using the statistical package SPSS Version 16 (Statistical Package for the Social Sciences, SPSS Inc, Chicago, IL, USA).

\section{Results}

Patients treated with PFNA were more likely $(p=0.002)$ to have impingement and fracture. In patients treated with PFNA II we identified none with impingement of the nail to the lateral cortex and none with lateral wall fragment or fracture displacement, whereas in patients treated with PFNA, we identified 10 occurrences of lateral impingement 
and in five of those 10 patients, the impingement resulted in fragmentation of the lateral wall. Fragmentation resulted in loss of reduction into varus in three of the five patients.

We observed fracture union in all PFNA II cases without mechanical failure complications. In PFNA cases, the fracture was united in 57 of 60 cases $(98.3 \%)$.

The operating time was shorter $(\mathrm{p}<0.001)$ in the PFNA II group compared with the PFNA group: 23 minutes versus 27 minutes, respectively; the difference likely has little if any clinical or economic importance.

PFNA II was comparable to PFNA regarding fluoroscopy time, need for transfusion, hospital stay, systemic complications, time to mobilization, and Harris hip score at 6 and 12 months (Table 3). We also found also no difference regarding fracture and operative variables such as preoperative mobility status, type of fracture, time to operation, quality of fixation, means, and quality of reduction (Table 2).

Both PFNA II and PFNA groups had three complications related to fixation (Table 3). In the PFNA group, two patients with intraarticular protrusion of the helical blade and one case of cutout were noticed (Fig. 4). In the PFNA II group, there were two patients with lack of compression of the fracture site initially and one case of breaking of the tip of the blade. Table 4 presents those complications and the way they were addressed.

\section{Discussion}

Proximal femoral nail antirotation, like other intramedullary devices for treating patients with unstable peritrochanteric fractures, has allowed a rapid minimally invasive approach with minimal surgical trauma and provided stable fixation allowing early mobilization with full weightbearing $[13,42]$. The major concern of this implant, however, is the impingement of the nail on the proximal lateral cortex that can cause lateral wall fracture and/or displacement of the reduced fracture. Synthes therefore designed the new version, the PFNA II, to overcome that concern.

Table 3. Outcome of evaluation

\begin{tabular}{llll}
\hline Outcome & PFNA & PFNA II & p value \\
\hline Time of operation, median (IQR) & $27.00(7.00)$ & $23.00(5.00)$ & $38.16 \pm 12.12$ \\
Fluoroscopy time, mean \pm SD & $41.79 \pm 15.12$ & $2.00(2.00)$ & 0.0005 \\
Time to mobilization, Median (IQR) & $2.00(1.00)$ & $81.92 \pm 7.68$ & 0.176 \\
Harris hip score at 6 months, mean \pm SD & $81.88 \pm 7.48$ & $81.42 \pm 9.00$ & 0.978 \\
Harris hip score at 12 months, mean \pm SD & $81.71 \pm 8.02$ & $4.00(1.00)$ & 0.861 \\
Hospital stay, median (IQR) & $4.00(1.00)$ & $1.74 \pm 1.00$ & 0.710 \\
Blood units transfused, mean \pm SD & $1.78 \pm 1.08$ & $50(100.0 \%)$ & 0.859 \\
Union (yes) & $57(98.3 \%)$ & $10(20 \%)$ & 1.000 \\
Systemic complications (yes) & $9(15.5 \%)$ & $3(6.0 \%)$ & 0.653 \\
Complications related to fixation (yes) & $3(5.2 \%)$ & 1.000 \\
\hline
\end{tabular}

PFNA = proximal femoral nail antirotation; IQR = interquartile range.
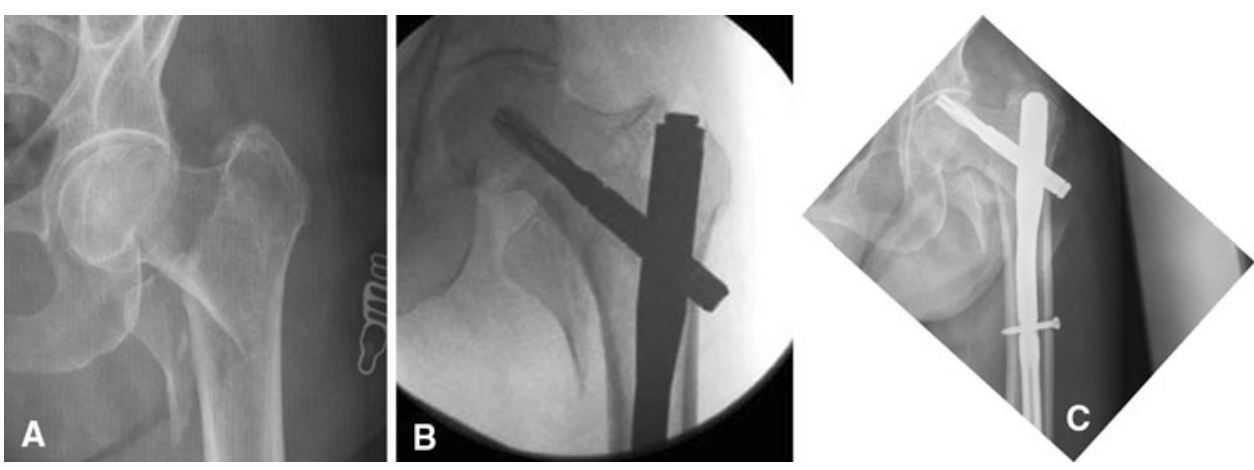

Fig. 4A-C (A) This AP radiograph illustrates an AO A2.2. unstable intertrochanteric fracture in a 72-year-old female patient. (B) PFNA was chosen for the treatment of patients in Figure 5A. After adequate reduction, the nail was inserted and the blade position was considered satisfactory. Note that the blade has not been locked. Locking of the blade was eventually forgotten. (C) This figure illustrates mechanical failure of the inappropriate fixation offered to the patient in $\mathrm{A}$ in the form of cutout of the blade that led to revision of the osteosynthesis. Technical steps must be strictly followed. 
Table 4. Complications related to fixation

\begin{tabular}{|c|c|c|}
\hline Complication & Number & Incident and treatment \\
\hline \multicolumn{3}{|l|}{ PFNA } \\
\hline $\begin{array}{l}\text { Intraarticular protrusion } \\
\text { of the helical blade }\end{array}$ & 2 & $\begin{array}{l}\text { Case 1: } 4 \text { months postoperatively; simple exchange } \\
\text { of the blade with a shorter one led } \\
\text { to an uneventful union } \\
\text { Case 2: } 8 \text { months postoperatively; patient remained } \\
\text { without weightbearing for } 6 \text { weeks and the helical blade } \\
\text { was removed, maintaining the intramedullary nail, } \\
\text { after union was secured }\end{array}$ \\
\hline Cutout & 1 & $\begin{array}{l}\text { Patient initially presented with an A2.2 AO fixation } \\
\text { (Fig. 4A); during surgery the helical blade was not } \\
\text { locked (Fig. 4B); patient presented at } 3 \text { months with cutout } \\
\text { of the blade (Fig. 4C); he was treated with revision } \\
\text { osteosynthesis using a } 95^{\circ} \text { dynamic plate } \\
\text { that led to uneventful union }\end{array}$ \\
\hline \multicolumn{3}{|l|}{ PFNA II } \\
\hline $\begin{array}{l}\text { Lack of compression of } \\
\text { the fracture site, initially }\end{array}$ & 2 & No consequences in fracture union \\
\hline $\begin{array}{l}\text { Breaking of the tip } \\
\text { of the blade }\end{array}$ & 1 & $\begin{array}{l}\text { Complication was a result of sharp hit and } \\
\text { malpositioning of the aiming arm; after appropriate } \\
\text { positioning of the instrumentation, the operation } \\
\text { was successfully completed }\end{array}$ \\
\hline
\end{tabular}

PFNA $=$ proximal femoral nail antirotation

Theoretically, the flattened lateral surface, together with the decreased mediolateral nail angle, prevents lateral wall impingement [39]. We therefore asked whether the PFNA II: (1) eliminates the lateral cortex impingement and fracture displacement experienced with PFNA; and (2) provides stable fracture fixation with a low major complication rate for unstable fractures in European patients.

A number of limitations of our study lessen the use of our observations. First, although a small study, we could identify fewer cases of lateral wall impingement with PFNA II compared with PFNA I. Second, our study was retrospective so the groups were not randomized and the PFNA I group was treated earlier than the PFNA II group. It is within the nature of implant evolution that newer implants are typically examined subsequent to existing implants. Third, peritrochanteric fractures may present with variable biomechanical differences, although we think it generally acceptable to treat these with intramedullary devices. One could argue that a comparison of intramedullary versus extramedullary devices for unstable peritrochanteric fractures could be included as a control group for the PFNA II device. Fourth, our data are those from a single surgeon and might not be generalizable. Finally, we found no major differences in the versatility of PFNA II compared with PFNA I; however, it appears that PFNA II better fits the proximal femur with fewer complications and thus with the numbers available, it appears the PFNA II is an improved design compared with PFNA I.
We found no patients with lateral cortex impingement in the PFNA II group but $10(17 \%)$ in the PFNA group. We further found impingement could lead to lateral fragment or fracture displacement, whereas absence of impingement prevented those complications during insertion. $\mathrm{Xu}$ et al. [42] in a comparative study of PFNA versus DHS in unstable peritrochanteric fractures reported two of 51 PFNA cases with minor split of the lateral cortex during insertion that were treated nonoperatively with subsequent uneventful union. Garg et al. [14] in a recent prospective study of PFNA versus DHS made no comment regarding difficulties or complications during nail insertion. Similarly, Gardenbroek et al. [13] in a retrospective comparison of PFNA versus PFN in unstable fractures favored the use of PFNA without commenting on lateral impingement or any of its sequences. Tyagi et al. [39] studied PFNA and PFNA II designs relative to proximal femoral anatomy in an Asian population using a CT-based analysis. Comparing proximal femoral anatomy with dimensions of PFNA and PFNA II, they concluded the lateral inclination angle and impingement length of femur are discrepant in PFNA; however, in PFNA II, the flat lateral surface reduced the risk of impingement with the lateral femoral cortex. Lv et al. [23] found PFNA II for unstable trochanteric fractures in Asian patients provided an anatomic fit in the proximal femur with $95 \%$ ideal position of the nail and eliminated complications related to fixation. The proximal femoral anatomy of European patients differs from that of 


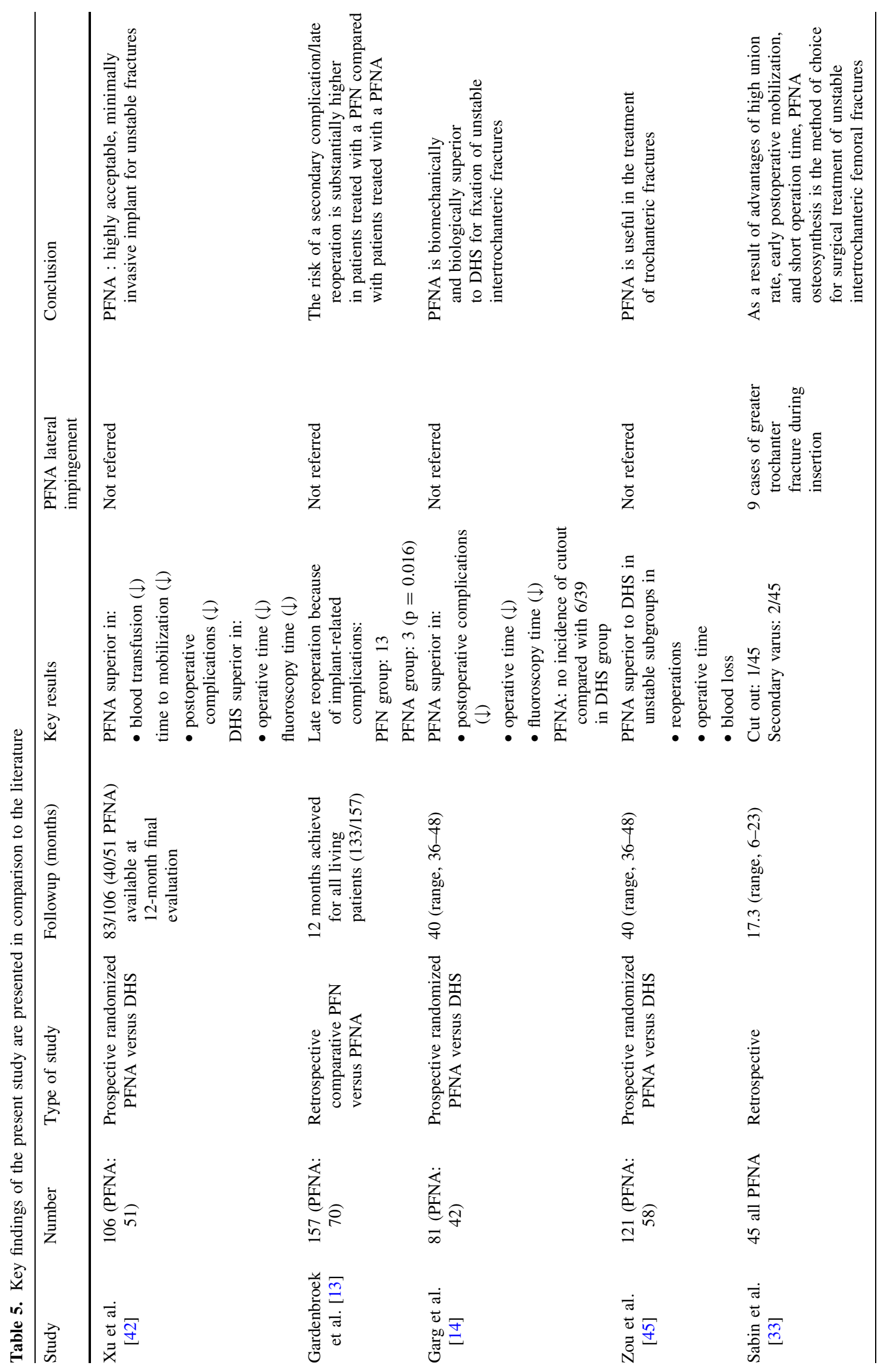


Asian people but we observed a rate of $17 \%$ lateral wall impingement among 58 patients treated with PFNA and none in 50 patients treated with PFNA II. Thus, PFNA II appears to reduce the risk of impingement.

Compared with PFNA, PFNA II was associated with a reduction in units of blood transfused, time to mobilization, hospital stay, complication rate, functional outcome, and higher rate of union. We observed no instance of cutout, no other major complication, and all fractures healed; only one patient had a reoperation for blade removal after the fracture had healed. Sporadic reports [11, 21] support the use of intramedullary implants instead of dynamic screws for treating peritrochanteric fractures in general and suggest there may be major benefits regarding rehabilitation services in the first 6 months after discharge and total expenditures for hospital services [4, 44]. However, other studies $[2,7,22,40]$ have concluded there is no clear benefit of cephalomedullary nailing versus extramedullary implants. It seems that intramedullary implants are beneficial in subtrochanteric and unstable fracture types [19, 31]. Among the latter are transverse or reverse oblique but also multifragmentary fractures that sustain intra- or postoperative shattering of the lateral trochanteric wall. Nails seem to prevent failure by opposing the uncontrollable medialization, and eventual failure, that occurs under these circumstances [18]. In the last 3 years, several studies [13, 14, 33, 36, 45] report high union rates combined with low major complication and reoperation rates and decreased blood loss and hospital stay with the use of PFNA in peritrochanteric fractures and especially unstable types (Table 5). Our observations confirm those in the literature $[13,14,33,36,45]$ suggesting PFNA is a reasonable option for unstable peritrochanteric fractures.

We found the operative time was slightly shorter (4 minutes, 23 versus 27 minutes) when using PFNA II compared with PFNA but this is likely of no clinical or economic importance. Mean fluoroscopy time was also shorter in PFNA II. The instrumentation of PFNA II was modified. The initial 17.0-mm drill bit is more flexible, whereas the handle is more easily removed by the static hexagonal socket. The small decrease in operative time could be attributed to the ease of insertion as a result of the flattened lateral nail surface and the decreased mediolateral angle that allows entrance at the tip of the greater trochanter with a reduced risk of trochanteric fragment or displacement. The recommended entry point of PFNA II is at the tip of the greater trochanter. Streubel et al. [37] suggested a high degree of variability exists for the ideal trochanteric entry site, yet the trochanteric tip represents the ideal starting point in only the minority of cases. They suggest an entry point $3 \mathrm{~mm}$ (mean value) medial to the tip as representative for most of the trochanteric nails. That differentiation with use of PFNA II compared with other techniques must be stressed. 
PFNA II minimizes the risk of lateral impingement encountered with PFNA. It can be easily inserted through the tip of the greater trochanter and be implanted fast, decreasing fluoroscopy time. The major complication rate was decreased without cutout or mechanical failure. We believe that this nail is a reasonable option and provides some advantages over the PFNA; at the same time, we emphasize that regardless of the implant choice and its specific technical characteristics, in the end, it is the technique of inserting it properly that is the key to succeed with stable fixation and prevent major complications. Currently PFNA II is our implant of choice in unstable peritrochanteric fractures.

\section{References}

1. Al-Yassari G, Langstaff RJ, Jones JWM, Al-Lami M. The AO/ ASIF proximal femoral nail (PFN) for the treatment of unstable trochanteric femoral fracture. Injury. 2002;33:395-399.

2. Anglen JO, Weinstein JN; American Board of Orthopaedic Surgery Research Committee. Nail or plate fixation of intertrochanteric hip fractures: changing pattern of practice. A review of the American Board of Orthopaedic Surgery Database. J Bone Joint Surg Am. 2008;90:700-707.

3. AO Foundation. Proximal Femur Nail Antirotation, PFNA®. Available at: www.aofoundation.org. Accessed June 2008.

4. Aros B, Tosteson AN, Gottlieb DJ, Koval KJ, Is a sliding hip screw or im nail the preferred implant for intertrochanteric fracture fixation? Clin Orthop Relat Res. 2008;466:2827-2832.

5. Banan H, Al-Sabti A, Jimulia T, Hart AJ. The treatment of unstable, extracapsular hip fractures with the AO/ASIF proximal femoral nail (PFN): our first 60 cases. Injury. 2002;33:401-405.

6. Baumgaertner MR, Curtin SL, Lindskog DM, Keggi JM. The value of the tip-apex distance in predicting failure of fixation of peritrochanteric fractures of the hip. J Bone Joint Surg Am. 1995;77:1058-1064.

7. Butler M, Forte ML, Joglekar SB, Swiontkowski MF, Kane RL. Evidence summary: systematic review of surgical treatments for geriatric hip fractures. J Bone Joint Surg Am. 2011;93:1104-1115.

8. Curtis MJ, Jinnah RH, Wilson V, Cunningham BW. Proximal femoral fractures: a biomechanical study to compare intramedullary and extramedullary fixation. Injury. 1994;25:99-104.

9. David A, von der HD, Pommer A. Therapeutic possibilities in trochanteric fractures. Safe-fast-stable. Orthopade. 2000;29:294-301.

10. Domingo LJ, Cecilia D, Herrera A, Resines C. Trochanteric fractures treated with a proximal femoral nail. Int Orthop. 2001;25:298-301.

11. Foulongne E, Gilleron M, Roussignol X, Lenoble E, Dujardin F. Mini-invasive nail versus DHS to fix pertrochanteric fractures: a case-control study. Injury. 2011;42:204-208.

12. Friedl W, Clausen J. [Experimental examination for optimized stabilisation of trochanteric femur fractures, intra- or extramedullary implant localisation and influence of femur neck component profile on cut-out risk] [in German]. Chirurg. 2001;72:1344-1352.

13. Gardenbroek TJ, Segers MJ, Simmermacher RK, Hammacher ER. The proximal femur nail antirotation: an identifiable improvement in the treatment of unstable pertrochanteric fractures? J Trauma. 2011;71:169-174.

14. Garg B, Marimuthu K, Kumar V, Malhotra R, Kotwal PP. Outcome of short proximal femoral nail antirotation and dynamic hip screw for fixation of unstable trochanteric fractures. A randomised prospective comparative trial. Hip Int. 2011;21:531-536.

15. Haidukewych GJ, Israel TA, Berry DJ. Reverse obliquity fractures of the intertrochanteric region of the femur. J Bone Joint Surg Am. 2001;83:643-650.

16. Harris WH. Traumatic arthritis of the hip after dislocation and acetabular fractures: treatment by mold arthroplasty. An endresult study using a new method of result evaluation. J Bone Joint Surg Am. 1969;51:737-755.

17. Kannus P, Parkkari J, Sievanen H, Heinonen A, Vuori I, Järvinen M. Epidemiology of hip fracture. Bone. 1996;18(Suppl):57S63S.

18. Kokoroghiannis C, Aktselis I, Deligeorgis A, Fragkomichalos E, Papadimas D, Pappadas I. Evolving concepts of stability and intramedullary fixation of intertrochanteric fractures-a review. Injury. 2012;43:686-693.

19. Kuzyk PR, Bhandari M, McKee MD, Russell TA, Schemitsch $\mathrm{EH}$. Intramedullary versus extramedullary fixation for subtrochanteric femur fractures J Orthop Trauma. 2009;23:465-470.

20. Laros GS. The role of osteoporosis in intertrochanteric fractures. Orthop Clin North Am. 1980;11:525-537.

21. Little NJ, Verma V, Fernando C, Elliott DS, Khaleel A. A prospective trial comparing the Holland nail with the dynamic hip screw in the treatment of intertrochanteric fractures of the hip. J Bone Joint Surg Br. 2008;90:1073-1078.

22. Liu M, Yang Z, Pei F, Huang F, Chen S, Xiang Z. A metaanalysis of the Gamma nail and dynamic hip screw in treating peritrochanteric fractures. Int Orthop. 2010;34:323-328.

23. Lv C, Fang Y, Liu L, Wang G, Yang T, Zhang H, Song Y. The new proximal femoral nail antirotation-Asia: early results. Orthopedics. 2011;34:351.

24. Madsen J, Næss L, Aune A, Alho A, Ekeland A, Strømsøe K. Dynamic hip screw with trochanteric stabilizing plate in the treatment of unstable proximal femoral fractures: a comparative study with the Gamma nail and compression hip screw. J Orthop Trauma. 1998;12:241-248.

25. Muller ME, Nazarian S, Koch P, Schatzker J (1990) The Comprehensive Classification of Fractures of Long Bones. 1st ed. Berlin, Heidelberg, Germany, New York, NY, USA: Springer-Verlag;

26. Orthopaedic Trauma Association Committee for Coding and Classification. Fracture and dislocation compendium. J Orthop Trauma. 1996;10(Suppl 1):v-ix, 1-154.

27. Pajarinen J, Lindahl J, Michelsson O, Savolainen V, Hirvensalo E. Pertrochanteric femoral fractures treated with a dynamic hip screw or a proximal femoral nail. A randomised study comparing postoperative rehabilitation. J Bone Joint Surg Br. 2005;87:76-81.

28. Panjabi MM, Lindsey RW, Walter SD, White AA 3rd. The clinician's ability to evaluate the strength of healing fractures from plain radiographs. J Orthop Trauma. 1989;3:29-32.

29. Parker MJ, Handoll HH. Gamma and other cephalocondylic intramedullary nails versus extramedullary implants for extracapsular hip fractures in adults. Cochrane Database Syst Rev. 2005;4:CD000093.

30. Parker MJ, Handoll HH. Intramedullary nails for extracapsular hip fractures in adults. Cochrane Database Syst Rev. 2006;3: CD004961.

31. Parker MJ, Handoll HH. Gamma and other cephalocondylic intramedullary nails versus extramedullary implants for extracapsular hip fractures in adults. Cochrane Database Syst Rev. 2010;9:CD000093.

32. Radford PJ, Needoff M, Webb JK. A prospective randomized comparison of the dynamic hip screw and the gamma locking nail. J Bone Joint Surg Br. 1993;75:789-793.

33. Sabin S, Ertürer E, Oztürk I, Toker S, Seçkin F, Akman S, Radiographic and functional results of osteosynthesis using the proximal femoral nail antirotation (PFNA) in the treatment of 
unstable intertrochanteric femoral fractures. Acta Orthop Traumatol Turc. 2010;44:127-134.

34. Schipper IB, Marti RK, van der Werken C. Unstable trochanteric femoral fractures: extramedullary or intramedullary fixation. Review of literature. Injury. 2004;35:142-151.

35. Sommers MB, Roth C, Hall H, Kam BC, Ehmke LW, Krieg JC, Madey SM, Bottlang M. A laboratory model to evaluate cutout resistance of implants for pertrochanteric fracture fixation. J Orthop Trauma. 2004;18:361-368.

36. Strauss E, Frank J, Lee J, Kummer FJ, Tejwani N. Helical blade versus sliding hip screw for treatment of unstable intertrochanteric hip fractures. A biomechanical evaluation. Injury. 2006; 37:984-989.

37. Streubel PN, Wong AH, Ricci WM, Gardner MJ. Is there a standard trochanteric entry site for nailing of subtrochanteric femur fractures? J Orthop Trauma. 2011;25:202-207.

38. Synthes. Proximal Femur Nail Antirotation, PFNA®. Available at: www.synthes.com. Accessed June 2008

39. Tyagi V, Yang JH, Oh KJ. A computed tomography-based analysis of proximal femoral geometry for lateral impingement with two types of proximal femoral nail anterotation in subtrochanteric fractures. Injury. 2010;41:857-861.
40. Verettas DA, Ifantidis P, Chatzipapas CN, Drosos GI, Xarchas KC, Chloropoulou P, Kazakos KI, Trypsianis G, Ververidis A. Systematic effects of surgical treatment of hip fractures: gliding screw-plating vs intramedullary nailing. Injury. 2010;41:279-284.

41. Windolf M, Braunstein V, Dutoit C, Schwieger K. Is a helical shaped implant a superior alternative to the dynamic hip screw for unstable femoral neck fractures? A biomechanical investigation. Clin Biomech (Bristol, Avon). 2009;24:59-64.

42. Xu YZ, Geng DC, Mao HQ, Zhu XS, Yang HL. A comparison of the proximal femoral nail antirotation device and dynamic hip screw in the treatment of unstable pertrochanteric fracture. $J$ Int Med Res. 2010;38:1266-1275.

43. Yoshimine F, Latta LL, Milne EL. Sliding characteristics of compression hip screws in the intertrochanteric fracture: a clinical study. J Orthop Trauma. 1993;7:348-353.

44. Ziran BH, Heckman DS, Olarte CM, Chou K, Baranick J. Intramedullary hip screw versus standard compression hip screw: early postoperative rehabilitation comparisons. Orthopedics. 2009; 32:83.

45. Zou J, Xu Y, Yang H. A comparison of proximal femoral nail antirotation and dynamic hip screw devices in trochanteric fractures. J Int Med Res. 2009;37:1057-1064. 